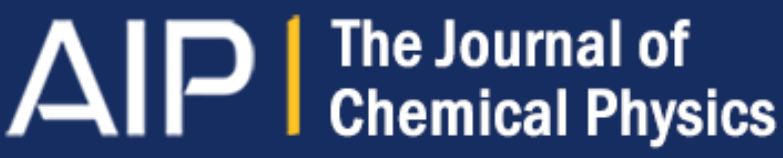

Communication: Reduced density matrices in molecular systems: Grand-canonical electron states

Roberto C. Bochicchio, Ramón A. Miranda-Quintana, and Diego Rial

Citation: The Journal of Chemical Physics 139, 191101 (2013); doi: 10.1063/1.4832495

View online: http://dx.doi.org/10.1063/1.4832495

View Table of Contents: http://scitation.aip.org/content/aip/journal/jcp/139/19?ver=pdfcov

Published by the AIP Publishing

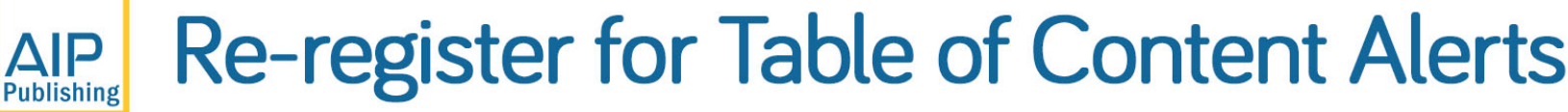




\title{
Communication: Reduced density matrices in molecular systems: Grand-canonical electron states
}

\author{
Roberto C. Bochicchio, ${ }^{1, a)}$ Ramón A. Miranda-Quintana, ${ }^{2}$ and Diego Rial ${ }^{3}$ \\ ${ }^{1}$ Departamento de Física, Facultad de Ciencias Exactas y Naturales, Universidad de Buenos Aires and IFIBA, \\ CONICET, Ciudad Universitaria, 1428 Buenos Aires, Argentina \\ ${ }^{2}$ Laboratory of Computational and Theoretical Chemistry, Faculty of Chemistry, University of Havana, \\ Zapata e G y Mazón, 10400 Havana, Cuba \\ ${ }^{3}$ Departamento de Matemática, Facultad de Ciencias Exactas y Naturales, Universidad de Buenos Aires \\ and IMAS, CONICET, Ciudad Universitaria, 1428 Buenos Aires, Argentina
}

(Received 20 September 2013; accepted 7 November 2013; published online 19 November 2013)

\begin{abstract}
Grand-canonical like descriptions of many electron atomic and molecular open systems which are characterized by a non-integer number of electrons are presented. Their associated reduced density matrices (RDMs) are obtained by introducing the contracting mapping for this type of distributions. It is shown that there is loss of information when connecting RDMs of different order by partial contractions. The energy convexity property of these systems simplifies the description. Consequently, this formulation opens the possibility to a new look for chemical descriptors such as chemical potential and reactivity among others. Examples are presented to discuss the theoretical aspects of this work. (C) 2013 AIP Publishing LLC. [http://dx.doi.org/10.1063/1.4832495]
\end{abstract}

The rearrangement of electron distributions in molecular systems under the influence of external perturbations, internal conversions, conformational changes, or reactive interactions is closely related to chemical reactivity which is relevant to understand the molecular structure. ${ }^{1-3}$ The chemical units involved in these processes are physical domains likewise, atoms, functional groups, or moieties within the molecular structure which exchange electrons between them, and possess a non-integer number of electrons interpreted as a time average in a quantum state of an open system. ${ }^{2}$ Their description is performed not only by means of the fundamental magnitudes like the energy and electron density, but also their derivatives ${ }^{1}$ which incorporate the changes in the number of particles and are at the very basis of the descriptor definitions. ${ }^{1}$ The states of such a systems can neither be described by a pure state nor for a canonical ensemble but by a statistical ensemble of pure states with different number of electrons, i.e., a grand-canonical like ensemble (GC). The density matrices (DMs) describing the quantum state contain the complete information about the system, i.e., from which all properties may be determined. ${ }^{4,5}$ Hence, the natural scenario for this description is that of the p-order reduced density matrices $(p-\mathrm{RDMs})^{6-8}$ in the GC framework, which are the quantum marginal distributions obtained from an average procedure over a subset of the variables of the DM, known as contraction mapping, ${ }^{8,9}$ and are simpler objects than the DM. ${ }^{8}$ The systems in which we are interested are atomic and molecular systems which exhibit a convex structure for their electronic energy ${ }^{2}$ and DM. ${ }^{10}$ In this Communication we introduce the contraction mapping for the GC description of the molecular open systems, and thus the $p$-RDMs and the energy of these systems are obtained within this framework. Also we

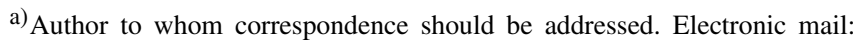
rboc@df.uba.ar
}

analyze their consequences and present some applicative examples to illustrate the theoretical results.

The density matrix $D$ describing the state of a system stands for a weighted sum of the complete set of all accessible $M$-electron pure state density matrices ${ }^{8}{ }^{M} D_{\Phi_{k}^{N}}=\left|\Phi_{k}^{M}\right\rangle\left\langle\Phi_{k}^{M}\right|$ in the mixture, where $\left|\Phi_{k}^{M}\right\rangle$ is the $k$ th quantum state function in the antisymmetric $M$-electron Hilbert space $\mathcal{F}_{M},{ }^{5,11}$ being its carrier space the entire Fock space $\mathcal{F}=\bigoplus_{M=0}^{\infty} \mathcal{F}_{M}$, where the $\bigoplus$ symbol indicates direct sum. ${ }^{11}$ Therefore, $D$ is expressed by

$$
\begin{aligned}
& D=\sum_{M} \sum_{\Phi_{k}^{M}} \omega_{\Phi_{k}^{M}}\left|\Phi_{k}^{M}\right\rangle\left\langle\Phi_{k}^{M}\right| ; \\
& \sum_{M} \sum_{\Phi_{k}^{M}} \omega_{\Phi_{k}^{M}}=1 ; \quad \omega_{\Phi_{k}^{M}} \geq 0,
\end{aligned}
$$

where $\omega_{\Phi_{k}^{M}}$ stands for the statistical weight, i.e., probability of occurrence of the pure $\left|\Phi_{k}^{M}\right\rangle$ state in the mixture. These states admit particle number fluctuation and hence the system may possess a non-integer number of particles. We will refer to it as the GC distribution. $D$ is a Hermitian, positive semi-definite (its eigenvalues are null or non-negative), bounded (the module of its elements is bounded), and finite trace (full real space integration) matrix, and because of its probabilistic interpretation it may be normalized to unity, i.e., $\operatorname{tr}(D)=\sum_{M} \sum_{\Phi_{k}^{M}} w_{\Phi_{k}^{M}}=1 .{ }^{4,5}$ Canonical distribution (C) (all states in the mixture possess the same number of particles $N$ ), expressed by ${ }^{N} D=\sum_{\Phi_{k}^{N}} \omega_{\Phi_{k}^{N}}\left|\Phi_{k}^{N}\right\rangle\left\langle\Phi_{k}^{N}\right|$ and the microcanonical (MC) distribution (all weights vanish except one), i.e., pure states ${ }^{N} D_{\Phi_{k}^{N}}=\left|\Phi_{k}^{N}\right\rangle\left\langle\Phi_{k}^{N}\right|$ are particular cases of the more general GC distribution.

The fundamental chemical concepts are the summary of the physical information contained in the $p$-RDMs of an $N$ electron molecular system $(p<N)$ which are derived from 
the DM and represent marginal distributions. ${ }^{8}$ Most of the attempts to describe the electron distribution in molecular systems have been concentrated on the 1-RDM $\left({ }^{1} D\right)$ and 2-RDM $\left({ }^{2} D\right)$, which determine electron densities and the energy of the system and provide intuitive interpretations of chemical data. ${ }^{1,3,12-14}$ Let us first sketch the contracting mapping (CM) for $\mathrm{C}$ and $\mathrm{MC}$ distributions as the basis to introduce this operation for the GC case. The CM, $\hat{L}_{p}^{N}$, applied to ${ }^{N} D^{8,9}$ gives rise to the $p$-RDM, i.e., ${ }^{p} D$, for both canonical and pure states. It stands for an averaging process over the remaining $N-p$ variables ${ }^{15}$ defined by

$$
\begin{aligned}
{ }^{p} D_{j_{1}, j_{2}, \ldots, j_{p}}^{i_{1}, i_{2}, \ldots, i_{p}} & =\left(\begin{array}{c}
N \\
p
\end{array}\right) \hat{L}_{p}^{N}\left\{{ }^{N} D\right\} \\
& =\sum_{\Phi_{k}^{N}} \omega_{\Phi_{k}^{N}}^{p} D_{j_{1}, j_{2}, \ldots, j_{p}}^{i_{1}, i_{2}, \ldots, i_{p}}\left(\Phi_{k}^{N}\right),
\end{aligned}
$$

where ${ }^{p} D_{j_{1}, j_{2}, \ldots, j_{p}}^{i_{1}, i_{2}, \ldots, i_{p}}\left(\Phi_{k}^{N}\right)=\left(\begin{array}{c}N \\ p\end{array}\right) \hat{L}_{p}^{N}\left\{{ }^{N} D_{\Phi_{k}^{N}}\right\}$ represents the $p$-RDM corresponding to the $\left|\Phi_{k}^{N}\right\rangle$ accessible pure state of the system and $\left(\begin{array}{c}N \\ p\end{array}\right)$ the combinatorial number for the Coleman normalization to the number of p-ons, i.e., number of composite $\mathrm{p}$-fermion particles, ${ }^{8}$ i.e., $p=1,2, \ldots$ stand for the one-electron reduced density matrix ${ }^{1} D$, the two-electron reduced density matrix ${ }^{2} D$, and so forth. The indices $i$, $j, \ldots$ denote a set of orthonormal one-electron functions (spinorbitals) which represent the basis set. The $p$-RDMs are hermitian, positive semi-definite, and bounded. ${ }^{6-8}$ Other essential property is its representability defined as the constraints that a given p-RDM must fulfill to be derivable from a DM corresponding to GC, C, or MC system state (cf. Eq. (1)). ${ }^{8,9}$ Let us remark that consequently for $\mathrm{C}$ and $\mathrm{MC}$ distributions in which the number of particles is fixed for all states in the distribution ${ }^{q} D$ and ${ }^{p} D(q<p)$ are connected by a contraction operation. ${ }^{8,9}$

The physical domains within the molecular structure mentioned above possessing a non-integer number of particles $\mathcal{N}=N+v$ with $N \in \mathbb{N}$ and $v \in \mathbb{R}$ in the interval $v \in(0,1)(\mathbb{N}$ and $\mathbb{R}$ fields of positive integer and real numbers, respectively) cannot be described either by a $\mathrm{C}$ or by a MC. Therefore, the appropriate scenario for that goal is the GC states (Eq. (1)), where the number of particles $M$ $(M \in \mathbb{N})$ is not fixed ${ }^{4,5}$ and then $\mathcal{N}$ may be considered as an average. ${ }^{2}$ This distribution is expressed by the DM in Fock space, ${ }^{5,11} D \in \mathcal{F}$ defined as $D=\bigoplus_{\left\{\Phi_{k}^{M}\right\}}^{\infty} \omega_{\Phi_{k}^{M}}{ }^{M} D_{\Phi_{k}^{M}}$, where ${ }^{M} D_{\Phi_{k}^{M}} \in \mathcal{F}_{M}, \omega_{\Phi_{k}^{M}} \geq 0$, and $\sum_{M \geq 0}^{\infty} \omega_{\Phi_{k}^{M}}=1$. The introduction of the CM for GC states to obtain the $p$-RDMs is performed by the application of the CM to $D$ given by Eq. (1) on the Fock space, hence it involves several states $\left|\Phi_{k}^{M}\right\rangle\left\langle\Phi_{k}^{M}\right|$, whose number of particles fulfill the condition that $M \geq p$, i.e., the order of contraction $p$ must be less than or equal to $M$. Thus, several states in the mixture may contribute to a $p$-RDM derived from a GC $D$, and all other states, whose $M<p$, will not contribute to this distribution. Namely, the states with $M<p$ do not contribute because they are not able to support p-distributions due to the number of particles they contain are less than the required to form the composed p-particles in the distribution, for instance, no pairs $(\mathrm{p}=2)$ may be formed from one particle systems, or 3-ons from two-particle sys- tems, and so on. Therefore, the $p$-RDMs for this ensemble may be defined by introducing the GC CM $\hat{L}_{p}$ by

$$
{ }^{p} D=\hat{L}_{p}\{D\}=\bigoplus_{\left\{\Phi_{k}^{M}, M \geq p\right\}} \omega_{\Phi_{k}^{M}}\left(\begin{array}{c}
M \\
p
\end{array}\right) \hat{L}_{p}^{M}\left\{{ }^{M} D_{\Phi_{k}^{M}}\right\},
$$

where it was assumed that $\hat{L}_{p}^{M}\left\{{ }^{M} D_{\Phi_{k}^{M}}\right\}=\mathbf{O}$ for $M<p$, and $\hat{L}_{p}^{p}\left\{{ }^{p} D_{\Phi_{k}^{p}}\right\}=\mathcal{I}$ with $\mathcal{I}$ and $\mathbf{O}$, the identity and null superoperators, respectively. ${ }^{16}$ These requirements complete the definition for the CM in Fock space. Therefore, the $p$-RDM is expressed by

$$
{ }^{p} D=\bigoplus_{\left\{\Phi_{k}^{M}, M \geq p\right\}} \omega_{\Phi_{k}^{M}}^{p} D_{\Phi_{k}^{M}}
$$

The trace operation is calculated by $\operatorname{Tr}\left({ }^{p} D\right)=\sum_{\left\{\Phi_{k}^{M}, M \geq p\right\}}$ $\omega_{\Phi_{k}^{M}}\left(\begin{array}{c}M \\ p\end{array}\right)=\left\langle\left(\begin{array}{c}M \\ p\end{array}\right)\right\rangle$, where $\langle\ldots\rangle$ indicates the average number of p-ons. For instance, for $p=1, \operatorname{Tr}\left({ }^{1} D\right)$ $=\sum_{\left\{\Phi_{k}^{M}, M \geq 1\right\}} \omega_{\Phi_{k}^{M}} M=\langle M\rangle$ is the average which can be expressed by a non-integer number $\langle M\rangle=N+v$, as stated above.

Some consequences arising from the reduced distributions in the $\mathrm{GC}$ framework are particularly interesting. In contrast with the $\mathrm{C}$ and $\mathrm{MC}$ states, in the GC distribution it is not possible to obtain ${ }^{q} D$ by contraction of a previously obtained ${ }^{p} D$ for $q<p^{8}$ without losing some information, hence such contraction may be performed only directly from $D$. It may be noted by expressing ${ }^{q} D$ as

$$
{ }^{q} D=\bigoplus_{\left\{\Phi_{k}^{M}, M \in[q, p)\right\}} \omega_{\Phi_{k}^{M}}^{q} D_{\Phi_{k}^{M}}+\bigoplus_{\left\{\Phi_{k}^{M}, M \geq p\right\}} \omega_{\Phi_{k}^{M}}{ }^{q} D_{\Phi_{k}^{M}},
$$

where the expression for ${ }^{q} D$ has been split into two contributions. The first term in Eq. (5) means that the number of particles of the $\left|\Phi_{k}^{M}\right\rangle$ states belong to the half-open interval $q \leq M<p$, i.e., $M \in[q, p)$, and the second term, the contributions from those states with $M \geq p$. This process represents symbolically the direct contraction operation $D \rightarrow{ }^{q} D$. Let us consider a contraction process performed in two steps with this previous one for comparison, i.e., to obtain ${ }^{p} D$ from $D$ and then to obtain ${ }^{q} D$ by ${ }^{p} D$ contraction, in symbols, $D \rightarrow{ }^{p} D \rightarrow{ }^{q} D$. It is clear that the difference between both contraction procedures to obtain ${ }^{q} D$ is described by the first term in Eq. (5). It is due to the fact that this term would not be present in ${ }^{p} D$ after $D$ contraction. Hence, it shows that the lost information distribution $I_{q, p}$, in performing the two step process, is $I_{q, p}=\bigoplus_{\left\{\Phi_{k}^{M}, M \epsilon[q, p)\right\}} \omega_{\Phi_{k}^{M}}{ }^{q} D_{\Phi_{k}^{M}}$ which indicates that the right marginal distributions within the GC distribution must be obtained applying the CM on $D$ matrix in a one step process. Nevertheless, for an interacting electron atomic or open molecular system driven by a 2-particle Coulombic potential with Hamiltonian, $\mathcal{H}=\sum_{i, j} h_{i j} c_{i}^{\dagger} c_{j}+\frac{1}{2} \sum_{i, j, k, l}\langle i j \mid k l\rangle c_{i}^{\dagger} c_{j}^{\dagger} c_{l} c_{k}$ with $c_{i}^{\dagger}$ and $c_{j}$ the standard creation/annihilation fermion operators, respectively, $h_{i j}$ the matrix elements of the mono-electronic terms $h$, and $\langle i j \mid k l\rangle$ those of the Coulomb bi-electronic terms of the Hamiltonian, ${ }^{17}$ the GC ground state DM possesses a convex structure ${ }^{10}$ expressed by

$$
D=(1-v){ }^{N} D_{0}+v{ }^{N+1} D_{0},
$$


where ${ }^{N} D_{0}$ and ${ }^{N+1} D_{0}$ stand for the $N$ and $N+1$ particle system DMs of a non-degenerate or removable degenerate ground state, respectively. ${ }^{18}$ Therefore, it is straightforward to see from Eqs. (5) and (6) that for this case there is no information loss, except for $q=N$ and $p=N+1$, i.e., for $q<N$, the ${ }^{q} D_{0}$ distribution can be equivalently obtained from $D$ or ${ }^{p} D_{0}(q<p)$. Let us see some interesting consequences from these results. The energy for a $M$-particle system may be expressed by ${ }^{19}$

$$
\mathcal{E}_{0}^{M}=\operatorname{Tr}\left({ }^{1} D_{o}^{M} h\right)+\operatorname{Tr}\left({ }^{2} D_{o}^{M} v\right)=\operatorname{Tr}\left({ }^{2} D_{o}^{M 2} \mathcal{K}_{M}\right)
$$

where ${ }^{1} D_{o}^{M}$ and ${ }^{2} D_{o}^{M}$ are the ground state 1- and 2-RDMs of the $M$-particle system as indicated by the supraindex $M$, or equivalently, by virtue of the reduced Hamiltonian ${ }^{2} \mathcal{K}_{M},{ }^{8,18} \mathcal{E}_{0}^{M}=\operatorname{Tr}\left({ }^{2} D_{o}^{M 2} \mathcal{K}_{M}\right)$ where its matrix elements are $\left({ }^{2} \mathcal{K}_{M}\right)_{k l}^{i j}=\frac{1}{M-1}\left(h_{i k} \delta_{j l}+h_{j l} \delta_{i k}\right)+\langle i j \mid k l\rangle$. Note that this operator depends on the number of particles. Before inspecting the form of the electronic energy of a non-integer number of particle systems let us introduce the extension of the uniqueness Rosina's theorem. ${ }^{18}$ For an interacting system this theorem establishes that the 2-RDM of a nondegenerate or removably degenerate ground state of an $M$ particle Hamiltonian with at most 2-particle interactions has a unique preimage. ${ }^{18}$ Then if each one of the elements of the set of ground states $\left\{\left|\Phi_{o}^{M}\right\rangle, M=1 \ldots \infty\right\}$ or equivalently their DMs $\left\{{ }^{M} D_{\Phi_{0}^{M}}, M=1 \ldots \infty\right\}$ are non-degenerate or removably degenerate states their associated 2-RDMs have a unique preimage, and therefore the corresponding one for the GC ensemble (cf. Eq. (4)) ${ }^{2} D=\bigoplus_{\left\{\Phi_{o}^{M}, M \geq 2\right\}} \omega_{\Phi_{o}^{M}}{ }^{2} D_{\Phi_{o}^{M}}$ is also unique. In particular, in atomic or molecular systems, contraction of $D$ in Eq. (6) leads to ${ }^{2} D=(1-v)^{2} D_{o}^{N}+v^{2} D_{o}^{N+1}$ and then the energy becomes

$$
\begin{aligned}
\mathcal{E}_{0}^{N+v}= & \operatorname{Tr}\left(\sum_{M=N}^{N+1} \omega_{M}{ }^{2} D_{o}^{M}{ }^{2} \mathcal{K}_{M}\right)=v \operatorname{Tr}\left({ }^{2} D_{o}^{N+1}{ }^{2} \mathcal{K}_{N+1}\right) \\
& +(1-v) \operatorname{Tr}\left({ }^{2} D_{o}^{N}{ }^{2} \mathcal{K}_{N}\right)
\end{aligned}
$$

or equivalently $\mathcal{E}_{0}^{N+v}=\operatorname{Tr}\left(D_{0}^{N+v} h\right)+\operatorname{Tr}\left({ }^{2} D_{0}^{N+v} v\right)$ which means that the energy is a functional $\mathbf{F}$ of ${ }^{2} D_{o}^{M}$, ${ }^{2} D_{o}^{M+1}$, and the fractional population number $v$, i.e., $\mathcal{E}_{0}^{N+v}$ $=\mathbf{F}\left({ }^{2} D_{o}^{N+1},{ }^{2} D_{o}^{N}, v\right)$.

We will present at this point some descriptive examples to illustrate the potentialities of the above theory. Let us begin at first considering a system in its ground state described at the Hartree-Fock level of approximation, whose GC DM (cf. Eq. (6)) reads

$$
\begin{aligned}
D= & (1-v)\left|\chi_{1} \ldots \chi_{N}\right\rangle\left\langle\chi_{1} \ldots \chi_{N}\right| \\
& +v\left|\chi_{1} \ldots \chi_{N} \chi_{N+1}\right\rangle\left\langle\chi_{1} \ldots \chi_{N} \chi_{N+1}\right|,
\end{aligned}
$$

where $\left\{\left|\chi_{i}\right\rangle, i=1,2, \ldots\right\}$ stands for the set of spin-orbitals in the Slater determinant states $\left|\chi_{1} \chi_{2} \ldots\right\rangle$ of the $N$ and $N+1$ electron systems assuming the frozen approximation. Then, performing the CM operation for $p=1$ (Eqs. (3) and
(6)), it yields

$$
\begin{aligned}
{ }^{1} D^{N+v}= & \hat{L}_{1}\{D\}=v\left(\begin{array}{c}
N+1 \\
1
\end{array}\right) \hat{L}_{1}^{N+1}\left({ }^{N+1} D_{0}\right) \\
& +(1-v)\left(\begin{array}{c}
N \\
1
\end{array}\right) \hat{L}_{1}^{N}\left({ }^{N} D_{0}\right),
\end{aligned}
$$

where $\quad\left(\begin{array}{c}N+1 \\ 1\end{array}\right) \hat{L}_{1}^{N+1}\left({ }^{N+1} D_{0}\right)={ }^{1} D_{0}^{N+1}$ and $\quad\left(\begin{array}{c}N \\ 1\end{array}\right) \hat{L}_{1}^{N}\left({ }^{N} D_{0}\right)$ $={ }^{1} D_{0}^{N}$. Hence, ${ }^{1} D^{N+v}=v^{1} D_{0}^{N+1}+(1-v){ }^{1} D_{0}^{N}$ stands for the 1-RDM of the $N+v$ particles system and introducing the structure of each ${ }^{1} D_{0}^{M_{7}}$ in Eq. (10) it follows

$$
{ }^{1} D_{0}^{N+v}=\sum_{i=1}^{N}\left|\chi_{i}\right\rangle\left\langle\chi_{i}|+v| \chi_{N+1}\right\rangle\left\langle\chi_{N+1}\right|,
$$

where the first term of the r.h.s. stands for the ${ }^{1} D_{0}^{N}$ and the remaining term, $v\left|\chi_{N+1}\right\rangle\left\langle\chi_{N+1}\right|$, is the single occupied spinorbital contribution from state of the $N+1$ system which is the fraction of the density difference between the states of $N+1$ and $N$ systems. Hence, it is immediate to obtain the energy for this system from Eq. (7) as

$$
E_{0}^{N+v}=E_{0}^{N}+v \varepsilon_{N+1},
$$

where $E_{0}^{N+v}$ and $E_{0}^{N}$ are the Hartree-Fock energies of the $N$ $+\nu$ - and $N$-particle systems, respectively, and $\varepsilon_{N+1}$ stands for $(N+1)$ th orbital energy. ${ }^{19}$ The same procedure may be followed for the case $N-v$ which yields $E_{0}^{N-v}=E_{0}^{N}$ $-v \varepsilon_{N}$. Thus, the energy is that of the $N$-particle system plus the $v$ fraction of the single occupied spin-orbital.

Other applications of importance are the determination of the first derivatives of the energy and the electron density with respect to the number of particles $\mathcal{N}$ at fixed external field $v$. These magnitudes are at the basis of the definitions of two fundamental chemical descriptors defined from the conceptual density functional theory: ${ }^{1,3}$ the chemical potential and the Fukui functions (reactivity indices), respectively. 1,3

The chemical potential becomes defined by

$$
\mu=\left(\frac{\partial \mathcal{E}_{o}^{\mathcal{N}}}{\partial \mathcal{N}}\right)_{v}
$$

regarding that the ground state energy of these open systems may be expressed by 2,10

$$
\mathcal{E}_{0}^{N \pm v}=(1-v) \mathcal{E}_{0}^{N}+v \mathcal{E}_{0}^{N \pm 1}
$$

with $\mathcal{E}_{0}^{N \pm v}, \mathcal{E}_{0}^{N}$, and $\mathcal{E}_{0}^{N \pm 1}$ the energy of the systems with $N \pm v, N$, and $N \pm 1$ electrons, respectively, and because $\partial \mathcal{N}= \pm \partial v(N=$ constant $)$, this derivative becomes

$$
\mu_{ \pm}= \pm\left(\frac{\partial \mathcal{E}_{o}^{\mathcal{N}}}{\partial v}\right)_{v}= \pm\left(\mathcal{E}_{0}^{N \pm 1}-\mathcal{E}_{0}^{N}\right)
$$

showing its discontinuity ${ }^{2}$ which yields

$$
\begin{aligned}
& \mu_{+}=\mathcal{E}_{0}^{N+1}-\mathcal{E}_{0}^{N}=-E A, \\
& \mu_{-}=\mathcal{E}_{0}^{N}-\mathcal{E}_{0}^{N-1}=-I P,
\end{aligned}
$$

where EA and IP stand for the electron affinity and the ionization potential, respectively. ${ }^{19}$ Therefore, the generalization 
of these formulae using Eq. (16) is obtained as $\left(\mathcal{E}_{o}^{N+v}-\mathcal{E}_{o}^{N}\right)$ $+\left(\mathcal{E}_{o}^{N-v}-\mathcal{E}_{o}^{N}\right)=I P^{N-v}-E A^{N+v}=v(I P-E A)$. Furthermore, the form of the Hartree-Fock energy (Eq. (12)) tempts to equate

$$
I P^{v}=v I P \text { and } E A^{v}=v E A
$$

where their physical meaning is based on the idea that on average $v$ electrons are removed or attached to the system, respectively.

The other important example is the first derivative of the electron density $\rho(\mathbf{r})$ at point $\mathbf{r}$ in space with respect to the number of particles at constant external field $v^{1,3}$ defined by $f^{ \pm}(\mathbf{r})=\left(\frac{\partial \rho(\mathbf{r})}{\partial \mathcal{N}}\right)_{v}^{ \pm}$. They are known as Fukui functions or reactivities. Following the same procedure as in the case of the energy, two derivatives arise regarding if $\mathcal{N}$ increases/decreases from $N$ to $N \pm v$, respectively, leading to $f^{ \pm}(\mathbf{r})= \pm\left(\frac{\partial \rho(\mathbf{r})}{\partial v}\right)_{v}^{ \pm}$. Therefore, as $\rho(\mathbf{r})$ is the diagonal element of ${ }^{1} D$ in the coordinate representation, ${ }^{8}$ these functions can be generalized to matrix form by $F^{ \pm}\left(\mathbf{r} \mid \mathbf{r}^{\prime}\right)= \pm\left(\frac{\partial}{\partial v}{ }^{1} D^{N \pm v}\left(\mathbf{r} \mid \mathbf{r}^{\prime}\right)\right)_{v}$ and introducing ${ }^{1} D^{N^{ \pm} v}$ it follows

$$
F^{ \pm}\left(\mathbf{r} \mid \mathbf{r}^{\prime}\right)= \pm\left({ }^{1} D^{N \pm 1}\left(\mathbf{r} \mid \mathbf{r}^{\prime}\right)-{ }^{1} D^{N}\left(\mathbf{r} \mid \mathbf{r}^{\prime}\right)\right) .
$$

This result represents a rigorous justification to the trial forms used to build accurate Fukui functions. ${ }^{20}$ In conclusion, the GC states to describe open molecular domains permit to introduce statistical concepts to describe electron distributions in the molecular structure even if they are few body systems. The first derivatives of the energy and electron density obtained from the GC states as fundamental chemical descriptors extend their definitions out of the density functional theory framework and thus these results are valid for any type of state functions, i.e., independent as well as correlated models. Note that following the same arguments as those applied in Ref. 21 , it is possible to obtain representations of $D$ with more than two terms but these are not states of minimal energy. ${ }^{10}$

This work is financially supported by Project Nos. 20020100100197, 20020090200242 (UBA, Argentina) and 11220090100061,11220090100637 (CONICET, Argentina).

${ }^{1}$ P. Geerlings, F. De Proft, and W. Langenaeker, Chem. Rev. 103, 1793 (2003).

${ }^{2}$ J. P. Perdew, R. G. Parr, M. Levy, and J. Balduz, Jr., Phys. Rev. Lett. 49, 1691 (1982).

${ }^{3}$ R. G. Parr and W. Yang, Density-Functional Theory of Atoms and Molecules (Oxford University Press, New York, 1989).

${ }^{4}$ D. Ter Haar, Rep. Prog. Phys. 24, 304 (1961).

${ }^{5} \mathrm{~K}$. Blum, Density Matrix Theory and Applications (Plenum, New York, 1981).

${ }^{6}$ P. O. Löwdin, Phys. Rev. 97, 1474 (1955).

${ }^{7}$ E. R. Davidson, Reduced Density Matrices in Quantum Chemistry (Academic, New York, 1976).

${ }^{8}$ A. J. Coleman and V. I. Yukalov, Reduced Density Matrices: Coulson's Challenge (Springer, New York, 2000).

${ }^{9}$ H. Kummer, J. Math. Phys. 8, 2063 (1967).

${ }^{10}$ R. C. Bochicchio and D. Rial, J. Chem. Phys. 137, 226101 (2012).

${ }^{11}$ G. G. Emch, Algebraic Methods in Statistical Mechanics and Quantum Field Theory (Wiley-Interscience, New York, 1972).

${ }^{12}$ R. C. Bochicchio, L. Lain, and A. Torre, Chem. Phys. Lett. 375, 45 (2003), and references therein.

${ }^{13}$ R. C. Bochicchio, J. Mol. Struct.: THEOCHEM 228, 209 (1991), and references therein.

${ }^{14}$ R. M. Lobayan and R. C. Bochicchio, Chem. Phys. Lett. 557, 154 (2013), and references therein.

${ }^{15}$ C. Valdemoro, Int. J. Quantum Chem. 60, 131 (1996).

${ }^{16}$ P. O. Löwdin, Int. J. Quantum Chem. 21, 275 (1982), and references therein.

${ }^{17}$ P. Surján, Second Quantized Approach to Quantum Chemistry (Springer, Berlin, 1989).

${ }^{18}$ D. A. Mazziotti, Phys. Rev. A 57, 4219 (1998).

${ }^{19}$ R. McWeeny, Methods of Molecular Quantum Mechanics (Academic, San Diego, 2001).

${ }^{20}$ P. Ayers and M. Levy, Theor. Chem. Acc. 103, 353 (2000).

${ }^{21}$ D. R. Alcoba, R. C. Bochicchio, G. E. Massacessi, L. Lain, and A. Torre, Phys. Rev. A 75, 012509 (2007). 